Supplementary Information

\title{
Systematic Quantification of Electron Transfer in a Bare Phospholipid Membrane using Nitroxide- Labelled Stearic Acids: Distance Dependence, Kinetics, and Activation Parameters
}

Max Schmallegger, ${ }^{\mathrm{a}}$ Antonio Barbon, ${ }^{* \mathrm{~b}}$ Marco Bortolus, ${ }^{\mathrm{b}}$ Angela Chemelli, ${ }^{\mathrm{c}}$ Itzhak Bilkis, ${ }^{\mathrm{d}}$ Georg Gescheidt*a and Lev Weiner $^{*} \mathrm{e}$

${ }^{a}$ Institute of Physical and Theoretical Chemistry, Graz University of Technology, Stremayrgasse 9, 8010 Graz, Austria

${ }^{\mathrm{b}}$ Dipartimento di Scienze Chimiche, Università degli Studi di Padova, Via Marzolo 1, 35131 Padova, Italy

${ }^{c}$ Institute of Inorganic Chemistry, Graz University of Technology, Stremayrgasse 9, 8010 Graz, Austria

${ }^{\mathrm{d}}$ Faculty of Agricultural, Food and Environmental Sciences, Hebrew University, 76100 Rehovot, Israel

${ }^{\mathrm{e}}$ Department of Chemical Research Support, Weizmann Institute of Science, 76100 Rehovot, Israel

Kinetic Model 2

Continuous-wave electron paramagnetic resonance 4

Time-resolved electron paramagnetic resonance 5

Small-angle X-ray scattering 6

Dynamic light scattering 6

$\begin{array}{ll}\text { Eyring Analysis } & 7\end{array}$

Kinetic parameters of the ET reaction within Marcus theory 8

EPR power saturation experiments in liposomes 11

References 12 


\section{Kinetic Model}

Scheme S1 displays the kinetic model used for the simulation of the cw-EPR decay traces: Here, $\mathrm{k}_{\text {exc }}$ is the rate of excitation and subsequent MLCT of $\left[\mathrm{Ru}(\mathrm{bpy})_{3}\right]^{2+}$ to $\left[\mathrm{Ru}(\mathrm{bpy})_{3}\right]^{2+*}\left(\mathrm{k}_{\mathrm{exc}} \geq 5.5 \mathrm{x}\right.$ $\left.10^{10} \mathrm{~s}^{-1}\right)^{1}$ and $\mathrm{k}_{\mathrm{ox}}$ is the rate of electron transfer between photo-excited $\left[\mathrm{Ru}(\mathrm{bpy})_{3}\right]^{2+*}$ residing in the aqueous phase outside the lipid bilayer and the sacrificial electron acceptor ammonium persulfate $\left(\mathrm{k}_{\mathrm{ox}}=1.2 \times 10^{9} \mathrm{M}^{-1} \mathrm{~s}^{-1}\right){ }^{2}$ We assume a complete and very fast conversion of the ground-state $\left[\mathrm{Ru}(\mathrm{bpy})_{3}\right]^{2+}$ to the photo-excited $\left[\mathrm{Ru}(\mathrm{bpy})_{3}\right]^{2+*}$ as we work under continuous irradiation. Importantly, we assume that the SNRs are distributed equally between the outer and inner leaflet of the lipid bilayer and do not undergo flip-flop motion during the time course of the simulation. This results in two distinctly different electron transfer rates $\left(\mathrm{k}_{\text {outer }}\right.$ and $\mathrm{k}_{\text {inner, }}$, marked in red and yellow, respectively), resulting in a (quasi) bi-exponential decay of the SNR intensity. The electron transfer rates $\left(\mathrm{k}_{\text {outer }}\right.$ and $\left.\mathrm{k}_{\text {inner }}\right)$ are evaluated by simulation of time-dependent decrease of the EPR signal of 5DSA and 16DSA nitroxides. (see Equations $1 \& 2$ in Scheme 3)

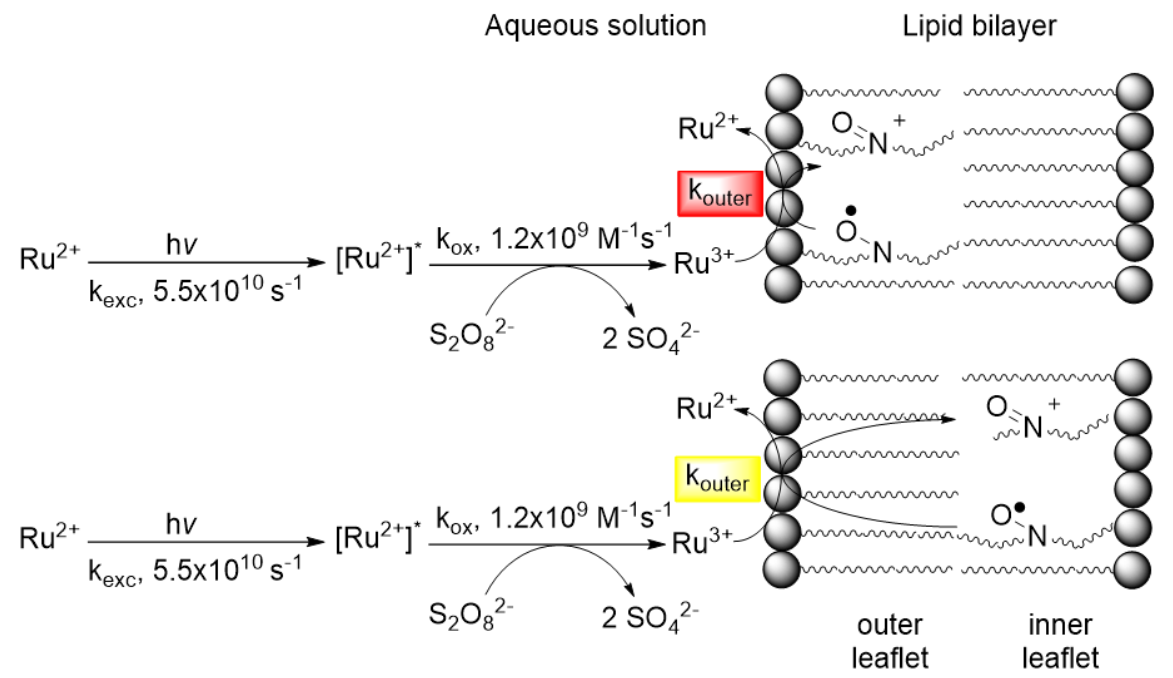

Scheme S1. Representation of the ET observed by cw-EPR, assuming two different distance regimes for electron transfer, yielding two distinctly different electron transfer rates $k_{\text {outer }}$ and $k_{\text {inner }}$ 
Scheme S2 displays the kinetic model used for the simulation of the TR-EPR decay traces. In this approach, the photo-excited $\left[\mathrm{Ru}(\mathrm{bpy})_{3}\right]^{2+}$ resides both outside the lipid bilayer and also in the internal aqueous volume of the liposome. Therefore, we only observe the fast electron transfer reaction ( $\mathrm{k}_{\text {outer, }}$ marked in red), resulting in a single decay component. Again, $\mathrm{k}_{\text {exc }}$ is the rate of excitation and MCLT and $\mathrm{k}_{\mathrm{exc}}$ is the rate of electron transfer between photo-excited $\left[\mathrm{Ru}(\mathrm{bpy})_{3}\right]^{2+*}$ and the sacrificial electron acceptor ammonium persulfate.

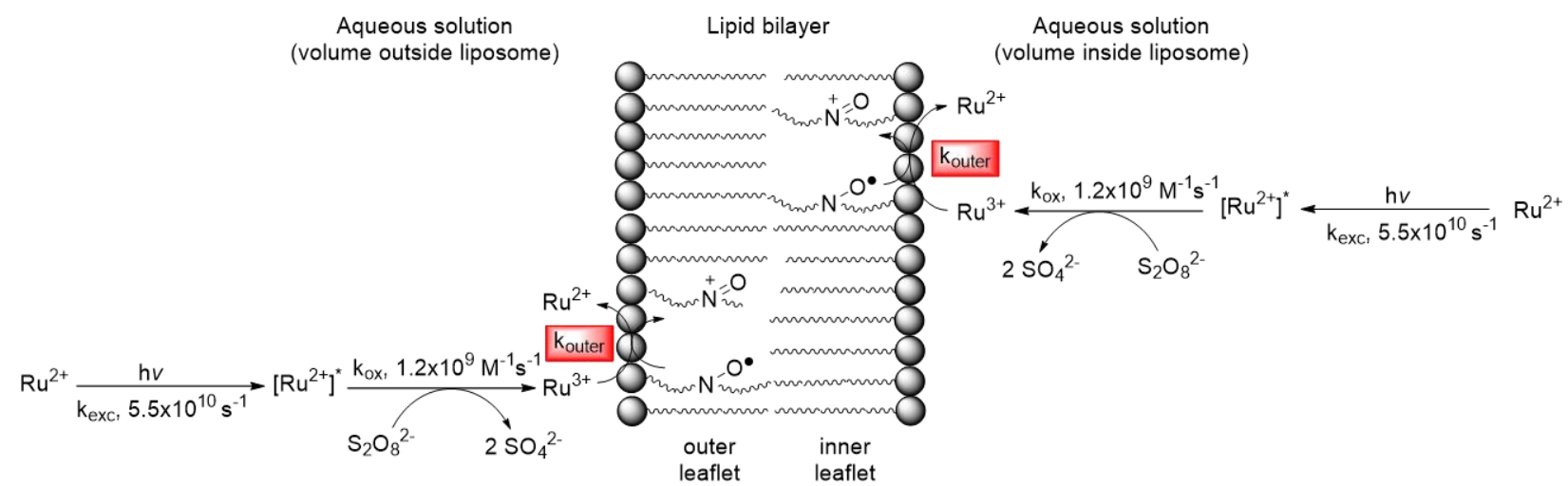

Scheme S2. Representation of the ET observed by TR-EPR; ruthenium complex is present both inside and outside the liposome yielding only the electron transfer rates $k_{\text {outer. }}$. 


\section{Continuous-wave electron paramagnetic resonance}

(correlation times and attempts of fitting of the signal decays)
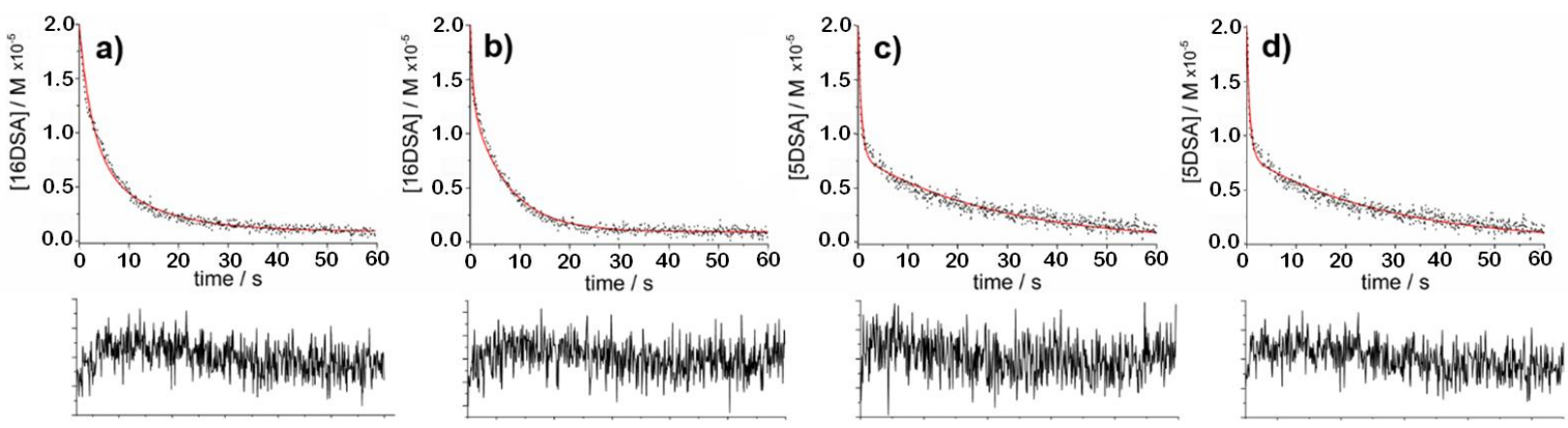

Figure S1. Experimental (black) and simulated (red) decay of the EPR intensity of 16DSA at $275 \mathrm{~K}(\mathrm{a})$ and $310 \mathrm{~K}(\mathrm{~b})$ and $\mathbf{5 D S A}$ at $275 \mathrm{~K}(\mathrm{c})$ and $310 \mathrm{~K}(\mathrm{~d})$ embedded into DMPC bilayer in the presence of [Ru(bpy) $\left.{ }_{3}\right] \mathrm{Cl}_{2}$ and $\left(\mathrm{NH}_{4}\right) \mathrm{S}_{2} \mathrm{O}_{8}$ upon continuous light irradiation; below the decay curves, the corresponding residuals of the fit (second order reaction) are shown
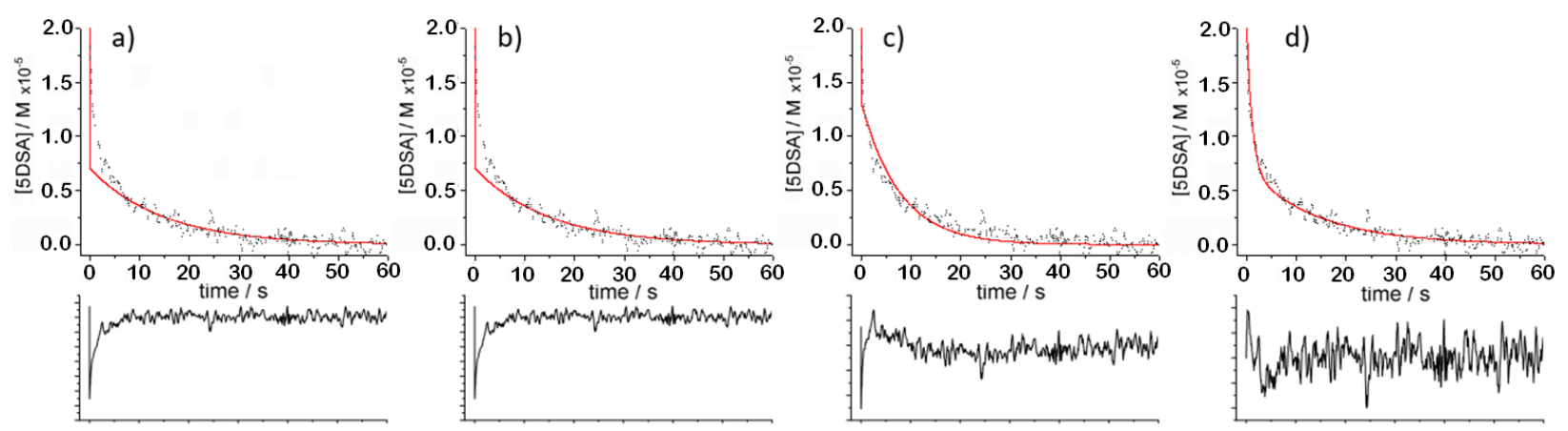

Figure S2. Experimental (black) and simulated (red) decay of the EPR intensity of 5DSA at 283 $\mathrm{K}$ embedded into DMPC bilayer in the presence of $\left[\mathrm{Ru}(\mathrm{bpy})_{3}\right] \mathrm{Cl}_{2}$ and $\left(\mathrm{NH}_{4}\right) \mathrm{S}_{2} \mathrm{O}_{8}$ upon continuous light irradiation with different kinetics models employed for the kinetic simulations; (a) first order kinetics for the fast and slow electron transfer (b) first order kinetics for the fast and second order kinetics for the slow electron transfer (c) second order kinetics for the fast and first order kinetics for the slow electron transfer (d) second order kinetics for the fast and slow electron transfer; below the decay curves, the corresponding residuals of the fits are shown 
Table S1. Correlation times of the EPR spectra of 16DSA and 5DSA embedded into a DMPC bilayer at different temperatures. The correlation times were calculated using the MATLAB based GUI SimLabel

\begin{tabular}{l|l|l}
\multirow{2}{*}{ Temperature $[\mathrm{K}]$} & Correlation Time $[\mathrm{s}]$ & \\
\cline { 2 - 3 } & 5DSA & 16DSA \\
\hline \hline 275 & $2.39 * 10^{-6}$ & $8.15 * 10^{-8}$ \\
\hline 283 & $3.60 * 10^{-6}$ & $3.20 * 10^{-9}$ \\
\hline 304 & $2.27 * 10^{-8}$ & $4.06 * 10^{-10}$ \\
\hline 310 & $5.89 * 10^{-9}$ & $6.51 * 10^{-10}$ \\
\hline \hline
\end{tabular}

Time-resolved electron paramagnetic resonance (attempts of fitting of the signal decays)
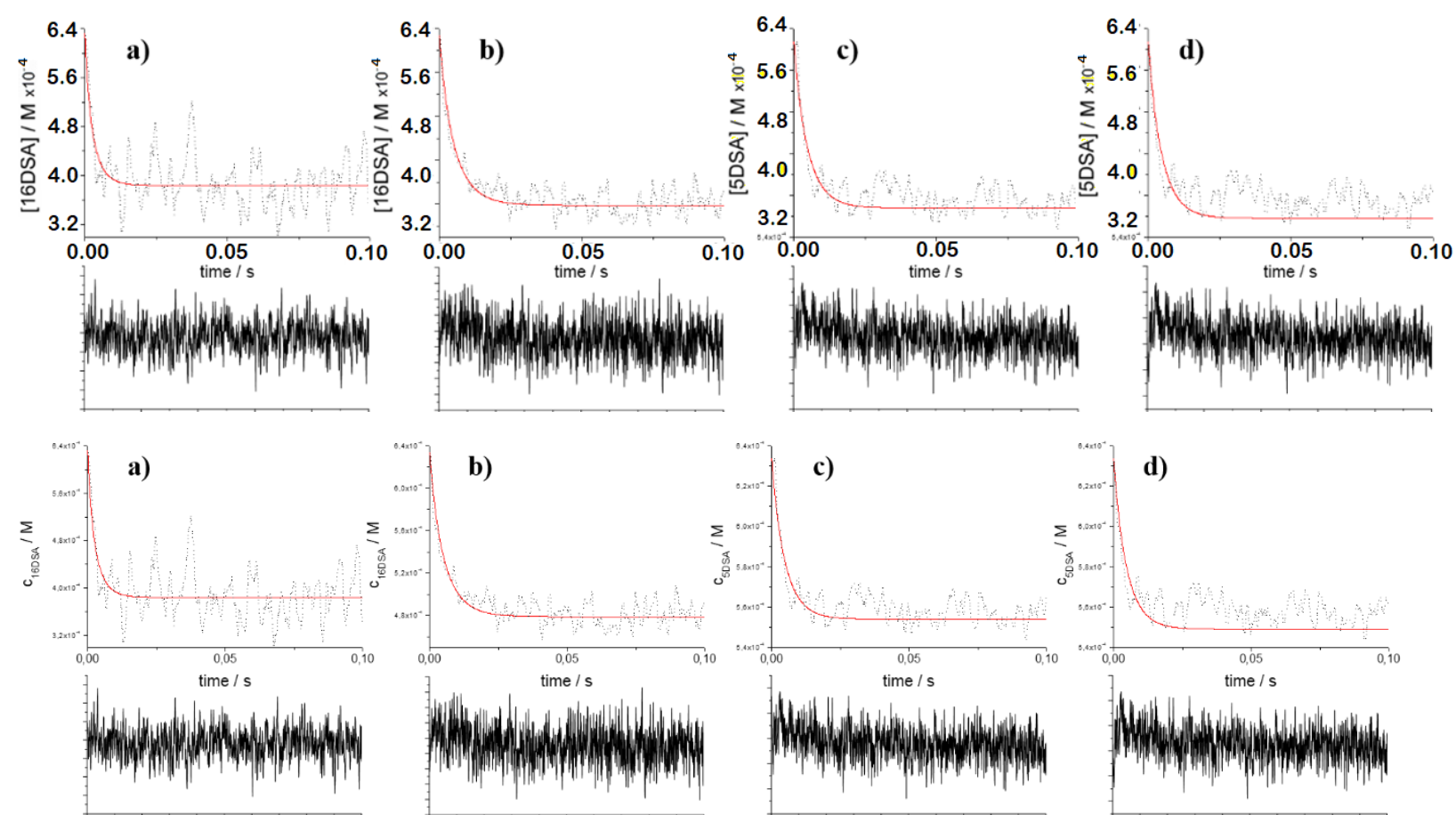

Figure S3. Experimental (black) and simulated (red) decay of the EPR intensity of 16DSA at $275 K($ a) and $310 K(b)$ and $\mathbf{5 D S A}$ at $275 K(c)$ and $310 K(d)$ embedded into DMPC bilayer in the presence of $\left[\mathrm{Ru}(\mathrm{bpy})_{3}\right] \mathrm{Cl}_{2}$ and $\left(\mathrm{NH}_{4}\right) \mathrm{S}_{2} \mathrm{O}_{8}$ upon pulsed laser irradiation; below the decay curves, the corresponding residuals of the fit (second order reaction) are shown 


\section{Small angle $X$-ray scattering}

The pair distance distribution functions of the liposomes reveal that the lipid bilayer for all three systems has a thickness of approx. $5.1 \mathrm{~nm}$ and that the incorporation of SNRs does not significantly alter the electron density of the lipid bilayers.

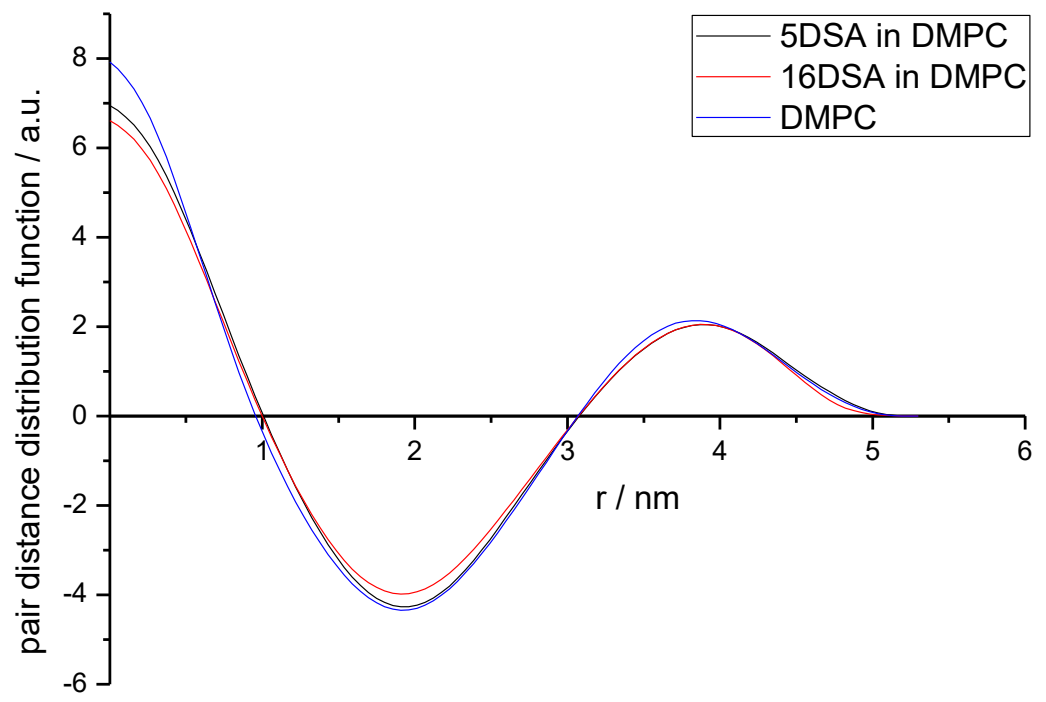

Figure S4. Pair-distance distribution of DMPC liposomes (blue) and DMPC liposomes incorporated with 16DSA (red) or 5DSA (blue) obtained by SAXS measurements

\section{Dynamic light scattering}
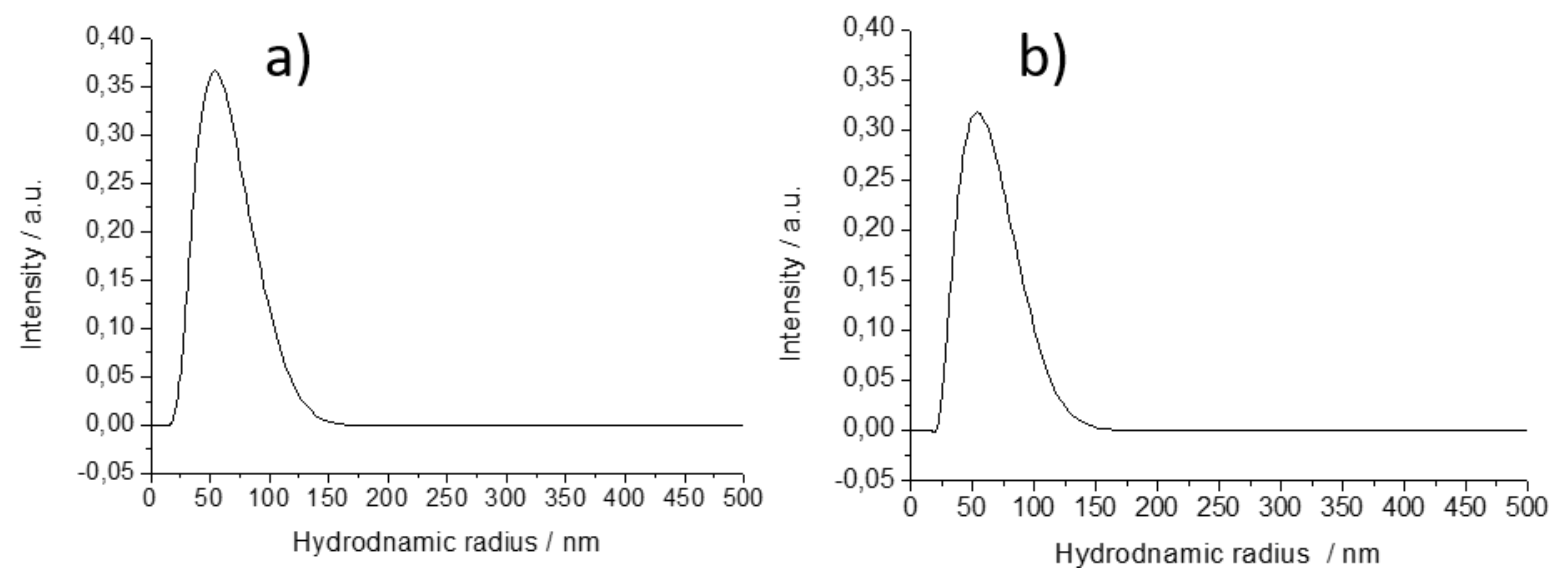

Figure S5. DLS measurements for DMPC liposomes with 16DSA (left) and 5DSA (right) incorporated into the lipid bilayer; both measurements show a narrow distribution of the liposome size centred around $53 \mathrm{~nm}$ 


\section{Eyring Analysis}

We evaluated the temperature-dependent rate constants determined for the fast oxidation of the nitroxyl moieties of 5DSA and 16DSA using Eyring theory (Equation S1): The corresponding plots and data are presented in Figure S6, and Table 2 in the manuscript.

$$
\ln \left(\frac{k_{E T}}{T}\right)=\left[\frac{\Delta S^{\ddagger}}{R}+\ln \left(\frac{k_{B}}{h}\right)\right]-\frac{\Delta H^{\ddagger}}{R T}
$$
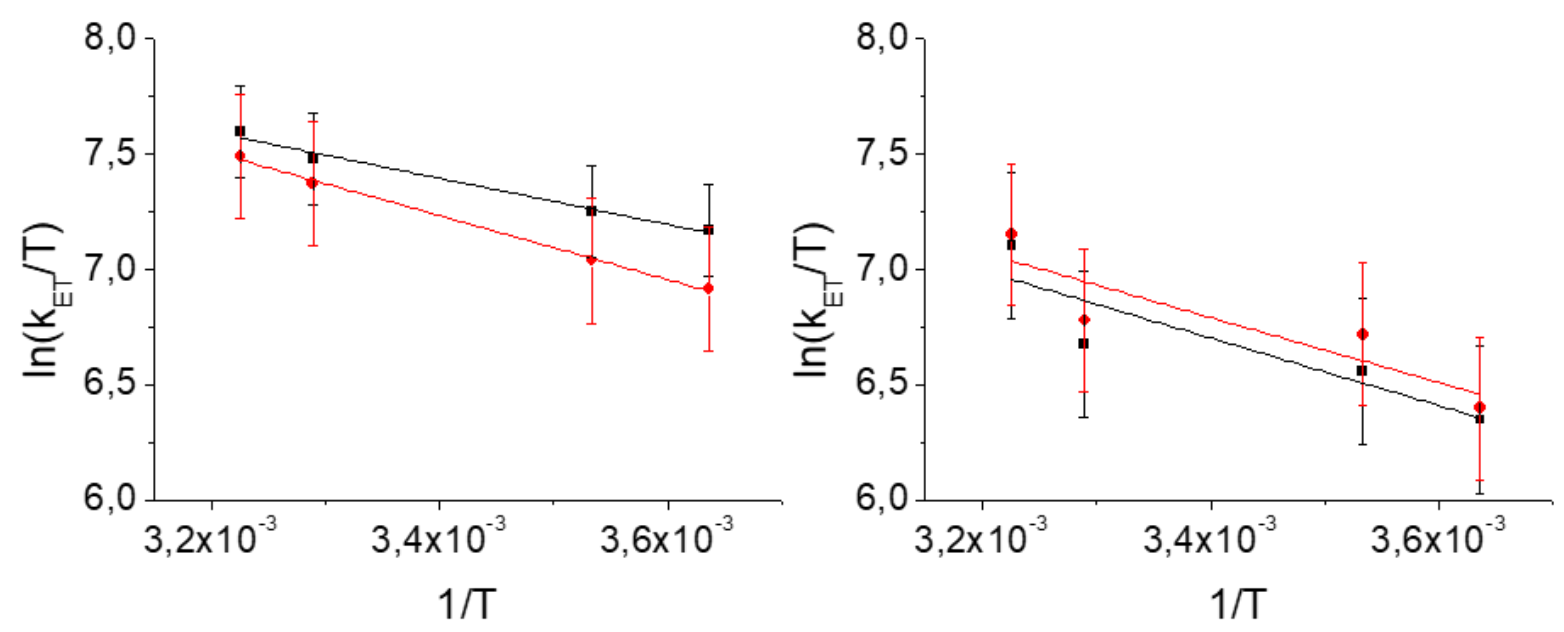

Figure S6. Eyring plots of the fast ET reaction for 5DSA (left) and 16DSA (right), measured by cw-EPR (black) and TR-EPR (red) 


\section{Kinetic parameters of ET reactions within Marcus theory}

\section{$\underline{\text { Reorganization energy calculation }}$}

According to Marcus theory, the activation free energy and the reorganization energy are connected via:

$$
\Delta G^{\ddagger}=\frac{\left(\lambda+\Delta G^{0}\right)^{2}}{4 \lambda k_{B} T}
$$

Here, $\Delta G^{0}$ is the reaction free energy. It was obtained from the redox potential of the $\left[\mathrm{Ru}(\mathrm{bpy})_{3}\right]^{2+} /\left[\mathrm{Ru}(\mathrm{bpy})_{3}\right]^{2+}$ pair $\left(1.30 \mathrm{~V}\right.$ vs. $\left.\mathrm{NHE}^{4}\right)$ and the nitroxide/N-oxoammonium pair $(0.9 \mathrm{~V}$ vs $\mathrm{NHE}^{5}$ ). The reorganization energy, $\lambda$, accounting for changes in solvation and molecular rearrangement following ET was determined from the equation S5 to be. $2.6 \pm 0.4 \mathrm{eV}$ for both SNRs. The corresponding activation free energies $\Delta G^{t}$ are reported in Table 2.

\section{Calculation of the Equilibrium Constant of Precursor Complex Formation K(r) and First-order} rate constants $k_{\text {Marcus }}$

Bimolecular electron transfer reactions are described in terms of two-step reactions: ${ }^{6}$ an encounter of the molecules to form a complex, and the ET within this complex. If the formation of the complex is not the rate determining step, the overall reaction is second-order. ${ }^{7}$ In our case, we can assume that the oxidant $\left([\mathrm{Ru}(\mathrm{bpy}) 3]^{3+}\right)$ is easily adsorbed at the phospholipid interface because of the opposite charge sign of the ion and the membrane, and it is linked to the SNR via a fatty acid "bridge". This assumption allows expressing the bimolecular rate constant $\mathrm{k}_{\mathrm{ET}}$ as the product of an equilibrium constant of the precursor complex $K(r)$ (in $\mathrm{M}^{-1}$ ) and a first-order rate constant $k_{\text {Marcus }}:^{8}$

$$
k_{E T}=K(r) k_{M a r c u s}
$$

with

$$
k_{\text {Marcus }}=\sqrt{\frac{4 \pi}{h^{2} \lambda k_{B} T}} H_{D A}^{2} \exp \left[-\frac{\Delta G^{\ddagger}}{k_{B} T}\right]
$$

and 


$$
K(r)=\frac{4 \pi N_{A} r^{2} \delta r}{1000} \exp \left(-\frac{w^{r}}{k_{B} T}\right)
$$

In this expression, $\mathrm{K}(\mathrm{r})$ is the equilibrium constant of the precursor complex $\mathrm{K}(\mathrm{r})$ (in $\left.\mathrm{M}^{-1}\right){ }^{9}$ The term $w^{r}$ is the work required to bring the reactants to the separation distance $r$, and $\delta r$ is the range of separation distances, over which the rate is appreciable. In our system, because of the favorable electrostatic interactions between the membrane surface and the oxidant ions, we assume a pre-equilibrium where the dissociation of the precursor complex is rapid compared to the rate of electron transfer within the precursor complex, and therefore, the electron transfer is not diffusion controlled. ${ }^{7}$ This is confirmed by the experimentally determined reaction order, confirming that the complex formation is much faster than the ET process. We assume that in our system $w^{r}$ is (almost) zero, since the separation distance $r$ between the reactants is mainly governed by the lipid bilayer. Therefore, the exponential term in equation S5 approaches 1 .

Employing equation S3 and S5 allows to estimating the equilibrium constants and the first-order ET rate constants $\mathrm{k}_{\text {Marcus: }}$ This estimation yields values of $\mathrm{K}(\mathrm{r})=4 \pm 1 \times 10^{1} \mathrm{M}^{-1}$ for 16DSA and $\mathrm{K}(\mathrm{r})=6 \pm 2 \times 10^{1} \mathrm{M}^{-1}$ for $\mathbf{5 D S A}$, allowing us to obtain values $\mathrm{k}_{\text {Marcus }}$ of $5 \pm 1 \times 10^{3} \mathrm{~s}^{-1}$ and $7 \pm 1 \mathrm{x}$ $10^{3} \mathrm{~s}^{-1}$ for 16DSA and 5DSA, respectively.

\section{Calculation of the Electronic Coupling Matrix $H_{A D}$ and of the Distance Decay Parameter $\square$}

Long-range electron tunnelling depends on the electronic factor $\left(H_{D A}\right)^{2}$ (see Equation S4). This term describes electronic coupling between distant donors and acceptors. ${ }^{10}$ The calculation of the electronic coupling matrix $H_{D A}$ was conducted using the estimated first-order ET rate constant $\mathrm{k}_{\text {Marcus }}$ (in $\mathrm{s}^{-1}$ ), according to equation $\mathrm{S} 4$. The values of the electronic coupling matrix element $H_{D A}$ amounts $9 \times 10^{-1} \pm 3 \times 10^{-1} \mathrm{~cm}^{-1}$ for 5DSA and 16DSA.

The distance dependence of electron tunnelling rates largely originates from the distance dependence of the electron coupling (see Equation S4). ${ }^{10}$ The electronic coupling $H_{D A}$ decreases exponentially with distance of donor-acceptor separation $(r)$ and the steepness of this decrease is expressed by the distance decay parameter $\beta$ (see Equation $\mathrm{S} 6$ ). ${ }^{11}$

$$
H_{D A}{ }^{2}={H_{D A}^{0}}^{2} \exp (-\beta \mathrm{r})
$$


Here, $\left(H_{D A}^{0}\right)^{2}$ is the electronic coupling when donor and acceptor in contact distance $(\mathrm{r}=0) \cdot{ }^{12,13}$ We estimated the distance decay parameter $\beta$ by plotting the rate constant $v s$. the distance of the nitroxyl moiety relative to $\mathrm{Ru}^{3+}$ residing in the outer aqueous phase for the various temperatures (see Figure S7). The temperature has a small effect on the slope (see the two linear fits, black lines, for the lower and the higher temperature). From the slope of the fitting line (red) it is possible to retrieve the $\beta$-value. We obtain a $\beta$-value for electron tunnelling through the phospholipid bilayer of $0.5 \pm 0.1 \mathrm{~nm}^{-1}$. This is comparable in magnitude to previously published values for distance decay parameters for phospholipid bilayers $\left(\beta=3.8 \mathrm{~nm}^{-1}\right)^{14}$. We think that one reason for the disagreement is mainly due to the definition of the distance. In ref 14 the measurement is conducted electrochemically, thus, likely, the technique is more sensitive to the apolar part than to the polar one. We considered geometric distances.

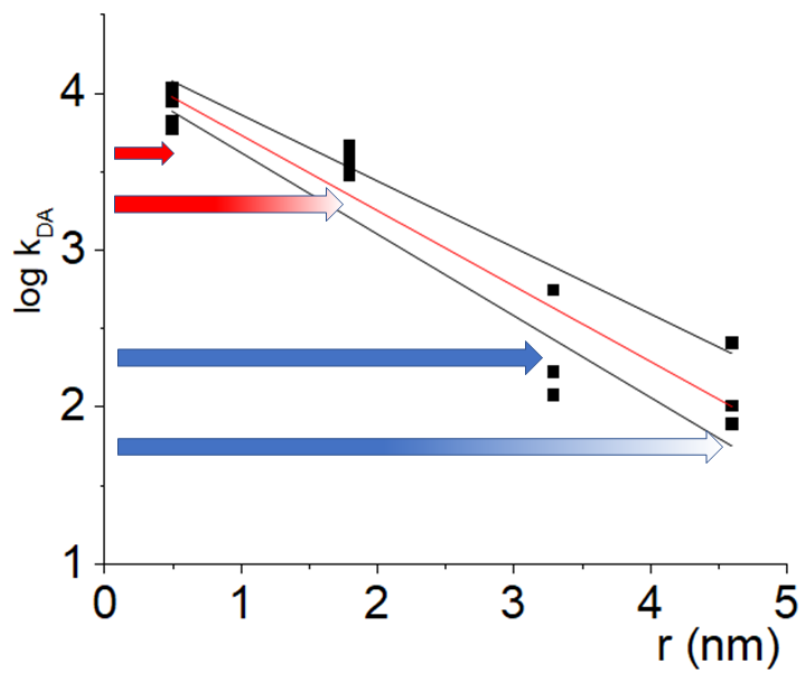

Figure S7. Plot of logarithms of the tunnelling-electron rate constants $k_{\text {Marcus }}$ versus the distance of separation $(r)$ of $\left[\mathrm{Ru}(\mathrm{bpy})_{3}\right]^{3+}$ and the nitroxyl moiety and its linear fit; the colour code of the arrows is the same as in Scheme 3.Black lines: linear fit relative to points at the same temperature. Red line: global linear fit. 


\section{EPR power saturation experiments in liposomes}

In Figure S8 below, we report the power saturation curves of 5DSA and 16DSA, with the fitting to obtain the $\mathrm{p}_{1 / 2}$ parameter according to the equation reported in the main text. In the insets, we report the spectra of the two labels in non-saturating conditions, $20 \mathrm{~mW}$. Note that the saturation experiment can be performed at room temperature, $293 \mathrm{~K}$ in our laboratory, just below the gelto-fluid transition, $296 \mathrm{~K}$ for pure DMPC.
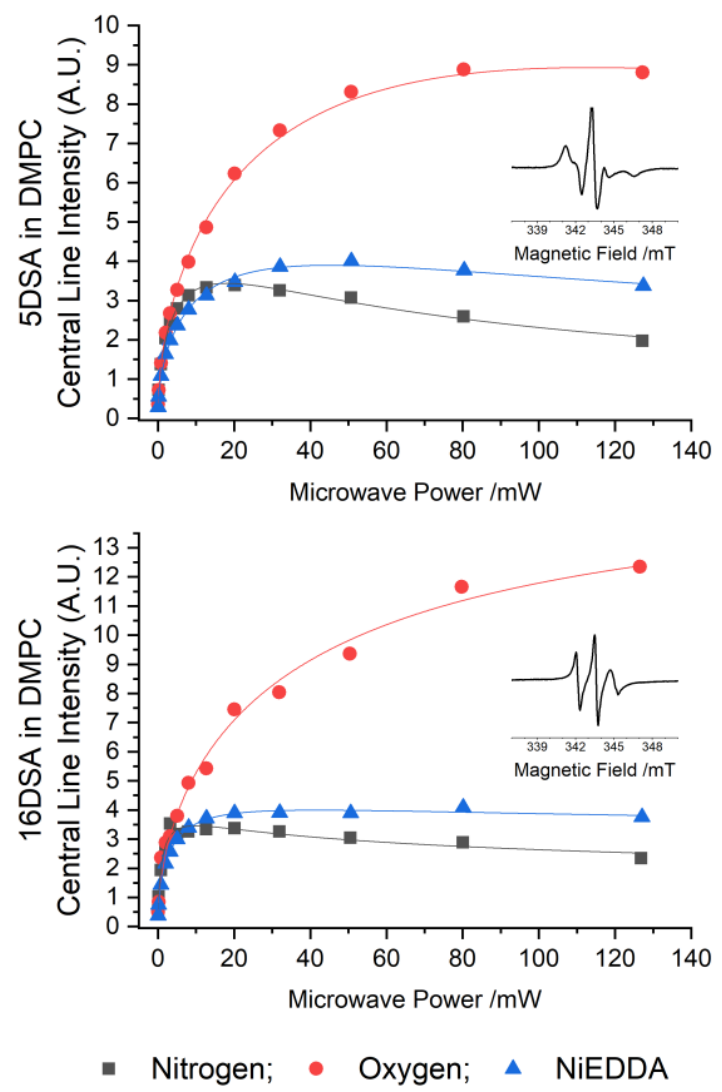

Figure S8. Doxyl-labeled fatty acid penetration depth in liposomes. Power saturation data (dots) and the fitting curves (lines) for 5DSA (top) and 16DSA (bottom) in DMPC liposomes, prepared in the same way as those for electron transfer experiments. The symbols indicate the different sample conditions: black squares, sample purged with nitrogen; red dots, sample in equilibrium with air (oxygen); blue triangles, sample with $20 \mathrm{mM} \mathrm{NiEDDA}$ and purged with nitrogen. In the insets the EPR spectra of the two spin probes. The experiments were performed at room temperature, $293 \mathrm{~K}$

In Table S2, we report the $\Phi$ parameter of the two SNRs compared to those of the standard nitroxide-labeled phospholipids (DPCs). From the comparison, we can see that 5DSA is close to 5DPC as penetration depth, just below the phosphates. 16DSA is located much deeper, close to the membrane center, at the same level as 14DPC. 
Table S2 Penetration depth parameter ( $\Phi$, adimensional) of the reference spin labels $(\boldsymbol{S L})$ in $P O P C^{15}$ and of 5DSA and 16DSA in DMPC liposomes obtained at room temperature.

\begin{tabular}{lccc}
\hline SL & $\Phi$ & Label & $\Phi$ \\
\hline 5DPC & $1.4^{\mathrm{a}}$ & 5DSA & 1.5 \\
7DPC & $1.2^{\mathrm{a}}$ & & \\
10DPC & 2.1 & & \\
12DPC & 2.4 & & 2.9 \\
14DPC & 3.0 & 16DSA & \\
${ }^{\mathrm{a}}$ An inversion in the linear trend was found also in the original work
\end{tabular}

\section{References}

(1) Ikeda, N.; Yoshimura, A.; Tsushima, M.; Ohno, T. Hopping and Annihilation of 3MLCT in the Crystalline Solid of $[\mathrm{Ru}(\mathrm{Bpy}) 3] \mathrm{X} 2(\mathrm{X}=\mathrm{Cl}-, \mathrm{ClO} 4-$ and PF6-). J. Phys. Chem. A 2000, 104 (26), 6158-6164.

(2) Horvath, A.; Bako, Z.; Papp, S. OXIDATIVE QUENCHING OF EXCITED Ru ( Bpy ), 2 + WITH S , 0g2- AT VARIOUS PH AND EXTERNAL MAGNETIC. J. Photochem. Photobiol. A Chem. 1990, 52, 271-280.

(3) Etienne, E.; Le Breton, N.; Martinho, M.; Mileo, M.; Belle, V. SimLabel: A Graphical User Interface to Simulate Contionous Wave EPR Spectra from Site-Directed Spin Labeling Experiments. Magn. Reson. Chem. 2017.

(4) Young, R. C.; Keene, F. R.; Meyer, T. J. Measurement of Rates of Electron Transfer between $\mathrm{Ru}(\mathrm{Bpy}) 33+$ and $\mathrm{Fe}(\mathrm{Phen}) 32+$ and between $\mathrm{Ru}(\mathrm{Phen}) 33+$ and $\mathrm{Ru}(\mathrm{Bpy}) 32+$ by Differential Excitation Flash Photolysis. J. Am. Chem. Soc. 1977, 99 (8), 2468-2473.

(5) Krishna, M. C.; Grahame, D. A.; Samuni, A.; Mitchell, J. B.; Krishna, M. C.; Grahamet, D. A.; Samunit, A.; Mitchell, J. B.; Russo, A. Oxoammonium Cation Intermediate in the Nitroxide-Catalyzed Dismutation of Superoxide. Proc. Natl. Acad. Sci. U. S. A. 1992, 89 (12), 5537-5541.

(6) Krishna, M. C.; Grahame, D. A.; Samuni, A.; Mitchell, J. B.; Russo, A. Oxoammonium Cation Intermediate in the Nitroxide-Catalyzed Dismutation of Superoxide. Proc. Natl. Acad. Sci. U. S. A. 1992, 89 (12), 5537-5541.

(7) Brown, G. M.; Sutin, N. A Comparison of the Rates of Electron Exchange Reactions of Ammine Complexes of Ruthenium(II) and-(III) with the Predictions of Adiabatic, OuterSphere Electron Transfer Models. J. Am. Chem. Soc. 1979, 101 (4), 883-892.

(8) Sutin, N. Theory of Electron Transfer Reactions. Prog. Inorg. Chem. 1983, 30, 441-498. 
(9) Marcus, R. A.; Sutin, N. Electron Transfers in Chemistry and Biology. BBA Rev.

Bioenerg. 1985, 811 (3), 265-322.

(10) Wenger, O. S. How Donor - Bridge - Acceptor Energetics Influence Electron Tunneling Dynamics and Their Distance Dependences. Acc. Chem. Res. 2011, 44 (1), 25-35.

(11) Paddon-Row, M. N. Investigating Long-Range Electron-Transfer Processes with Rigid, Covalently Linked Donor-(Norbornylogous Bridge)-Acceptor Systems. Acc. Chem. Res. 1994, 27 (1), 18-25.

(12) Eng, M. P.; Albinsson, B. Non-Exponential Distance Dependence of Bridge-Mediated Electronic Coupling. Angew. Chemie - Int. Ed. 2006, 45 (34), 5626-5629.

(13) Williams, R. M. Distance and Orientation Dependence of Photoinduced Electron Transfer through Twisted, Bent and Helical Bridges: A Karplus Relation for Charge Transfer Interaction. Photochem. Photobiol. Sci. 2010, 9 (7), 1018-1026.

(14) Campos, R.; Kataky, R. Electron Transport in Supported and Tethered Lipid Bilayers Modified with Bioelectroactive Molecules. J. Phys. Chem. B 2012, 116 (13), 3909-3917.

(15) Bortolus, M.; Dalzini, A.; Formaggio, F.; Toniolo, C.; Gobbo, M.; Maniero, A. L. An EPR Study of Ampullosporin A, a Medium-Length Peptaibiotic, in Bicelles and Vesicles. Phys. Chem. Chem. Phys. 2016, 18 (2), 749-760.

(16) Altenbach, C.; Greenhalgh, D. A.; Khorana, H. G.; Hubbell, W. L. A Collision Gradient Method to Determine the Immersion Depth of Nitroxides in Lipid Bilayers: Application to Spin-Labeled Mutants of Bacteriorhodopsin. Proc. Natl. Acad. Sci. 2006, 91 (5), 16671671. 